\title{
Enhanced magnetoresistance in layered magnetic structures with antiferromagnetic interlayer exchange
}

\author{
G. Binasch, P. Grünberg, F. Saurenbach, and W. Zinn \\ Institut für Festkörperforschung, Kernforschungsanlage Jülich G.m.b.H., Postfach 1913, D-5170 Jülich, West Germany
}

(Received 31 May 1988; revised manuscript received 12 December 1988)

\begin{abstract}
The electrical resistivity of $\mathrm{Fe}-\mathrm{Cr}-\mathrm{Fe}$ layers with antiferromagnetic interlayer exchange increases when the magnetizations of the Fe layers are aligned antiparallel. The effect is much stronger than the usual anisotropic magnetoresistance and further increases in structures with more than two Fe layers. It can be explained in terms of spin-flip scattering of conduction electrons caused by the antiparallel alignment of the magnetization.
\end{abstract}

Currently there is much interest in layered magnetic structures, which is partly due to the prospect that layering can be used to modify the material properties or to obtain new properties, uncharacteristic for the bulk materials. In the past few years we have concentrated our research on exploration of the exchange coupling between different magnetic films and on the coupling of ferromagnetic films across nonmagnetic or antiferromagnetic interlayers. For practical reasons we have restricted the work to the most simple structure where this question can be investigated, i.e., a magnetic double layer consisting of two ferromagnetic films interspaced by a film of another material. A very interesting case which we found during the course of this work was double layers of Fe interspaced by $\mathrm{Cr}$ as sketched in Fig. 1. If these films are of reasonably good monocrystalline quality and if the thickness $d_{0}$ of the Cr film is approximately $1 \mathrm{~nm}$, then we observed that the effective exchange coupling of the Fe layers across the $\mathrm{Cr}$ is antiferromagnetic (AF). This happens for epitaxial growth of the layered $\mathrm{Fe}-\mathrm{Cr}-\mathrm{Fe}$ structure both along the [100] and [110] crystallographic directions. ${ }^{1-3}$

Although the microscopic origin of this AF coupling up to now remains somewhat unclear, we found that such structures display some novel and unique magnetic properties both in their static and dynamic behavior. ${ }^{1-3}$ The new feature we report on here and which also comes as a result of the AF coupling is a strong increase of the magnetoresistance effect. Usually magnetoresistance refers to the so-called anisotropic effect, i.e., the difference in resistivity, $\Delta R=R_{\perp}-R_{\|}$for currents flowing parallel $\left(R_{\|}\right)$and perpendicular $\left(R_{\perp}\right)$ to the magnetization. As we show here, in layered structures with AF coupling a change in resistivity due to antiparallel alignment of the magnetizations in the ferromagnetic films can be observed. In the investigated cases it is much stronger than the anisotropic effect. It is clear that this is an attractive aspect for applications, such as magnetoresistive field sensors.

We have two methods available to recognize AF coupling, namely hysteresis curves measured via the magneto-optic Kerr effect (MOKE) and light scattering (LS) from spin waves. A more extensive description has been given elsewhere. ${ }^{4}$ Here we will exploit the peculiar behavior of spin waves in the antiparallel aligned state as shown in Fig. 1. The spectra we show can be obtained only in this state and therefore can be used as a signature of it. The scattering geometry is also of importance because the observed waves have to propagate perpendicular to the sample magnetization $J$. Since the propagation direction is determined by the plane of incidence of the probing laser light this fact can be used to determine the direction of $J$. The direction of the externally applied field $B_{0}$, of course, is known. We will encounter two important cases: $J$ is collinear with $B_{0}$ in the one and perpendicular to it in the other.

In order to be able to measure magnetoresistance of such samples, they were made in the shape of thin strips. The strip width was $1 \mathrm{~mm}$, with a length of $10 \mathrm{~mm}$. This is large enough to focus a laser beam onto the sample, which is necessary to apply the methods described above.

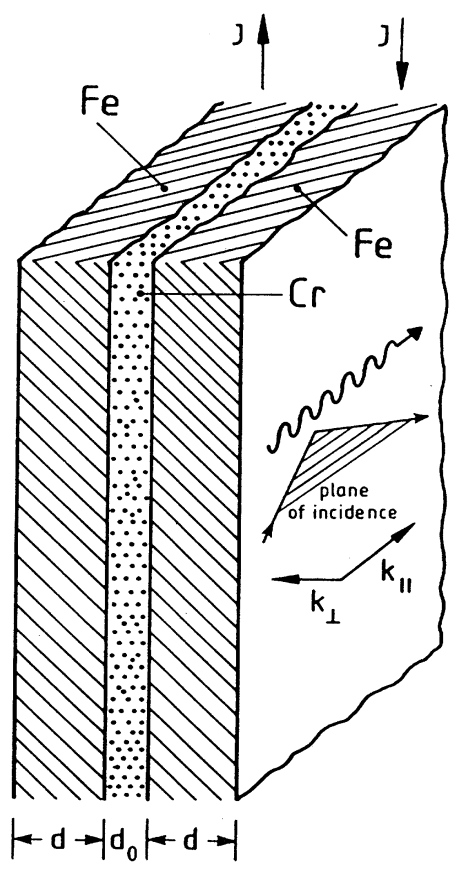

FIG. 1. Ferromagnetic double layer with antiparallel alignment of the magnetizations. Also indicated is the plane of incidence of the laser light for the observation of light scattering from spin waves and hysteresis curves via MOKE. 
Resistance was measured with the usual four-point method with current and voltage leads on both ends of the strip. The samples were grown epitaxially on [110]oriented $\mathrm{GaAs}$ by the well-established method ${ }^{5}$ and hence the film plane is parallel to a (110) atomic plane. For the thickness $d$ of the individual Fe films, we chose $d=12 \mathrm{~nm}$ and confirmed that the easy axis (EA) was along [100]. For smaller values of $d$ one has to be careful because the EA can switch to a [110] direction. ${ }^{6}$ In our case the [110] direction was the in-plane hard axis (HA). The long axis of the strip was parallel to a [100] direction and hence the EA of the sample. The $\mathrm{Cr}$ thickness was $d_{0}=1 \mathrm{~nm}$, which leads to AF coupling in agreement with previous results. $^{2,3}$ As a reference sample we also made a single $\mathrm{Fe}$ film with thickness $d=25 \mathrm{~nm}$ in order to measure, for comparison only, the anisotropic magnetoresistance (MR) effect. Morphology and composition during growth of the samples were monitored by means of spin-polarized lowenergy electron-diffraction and Auger analysis.

In Figs. 2(a) and 2(b) we see the MOKE hysteresis loops from the double layers with AF coupling for $B_{0}$ along the EA and HA. The directions of the magnetization are indicated by the encircled pairs of arrows. This information is obtained from the MOKE intensities and the displayed LS spectra. Let us discuss as an example the hysteresis loop shown in Fig. 2(a) in more detail. The field $B_{0}$ is applied along the EA, which is the long axis of the strip. It is clear that for large enough $B_{0}$ the samples saturate in the field direction (parallel alignment). If we start with parallel alignment in the positive field direction and reduce $B_{0}$ then at a certain, but still positive value of $B_{0}$, the magnetization of one film reverses via domain-wall motion (point 1). Hence in small fields we have antiparallel alignment. In a negative field, at point 2 , the other film also reverses, and we have saturation. Points 3 and 4 mark the magnetization reversals when $B_{0}$ is scanned back. From the size of the MOKE signal at points 1-4 one learns which of the two films reverses the magnetization. The larger change is due to the upper film. We see that in Fig. 2(a) the lower film always reverses first, independent of the direction of the field scan. We also had samples where the upper film always reversed first. Obviously, this is caused by slightly different coercive fields of the two Fe films. In the low-field regime, light scattering from spin waves has been performed and the spectra are also displayed. A typical feature of these spectra is the fact that Stokes and anti-Stokes scattering is observed at different frequencies. As has been explained in more de-
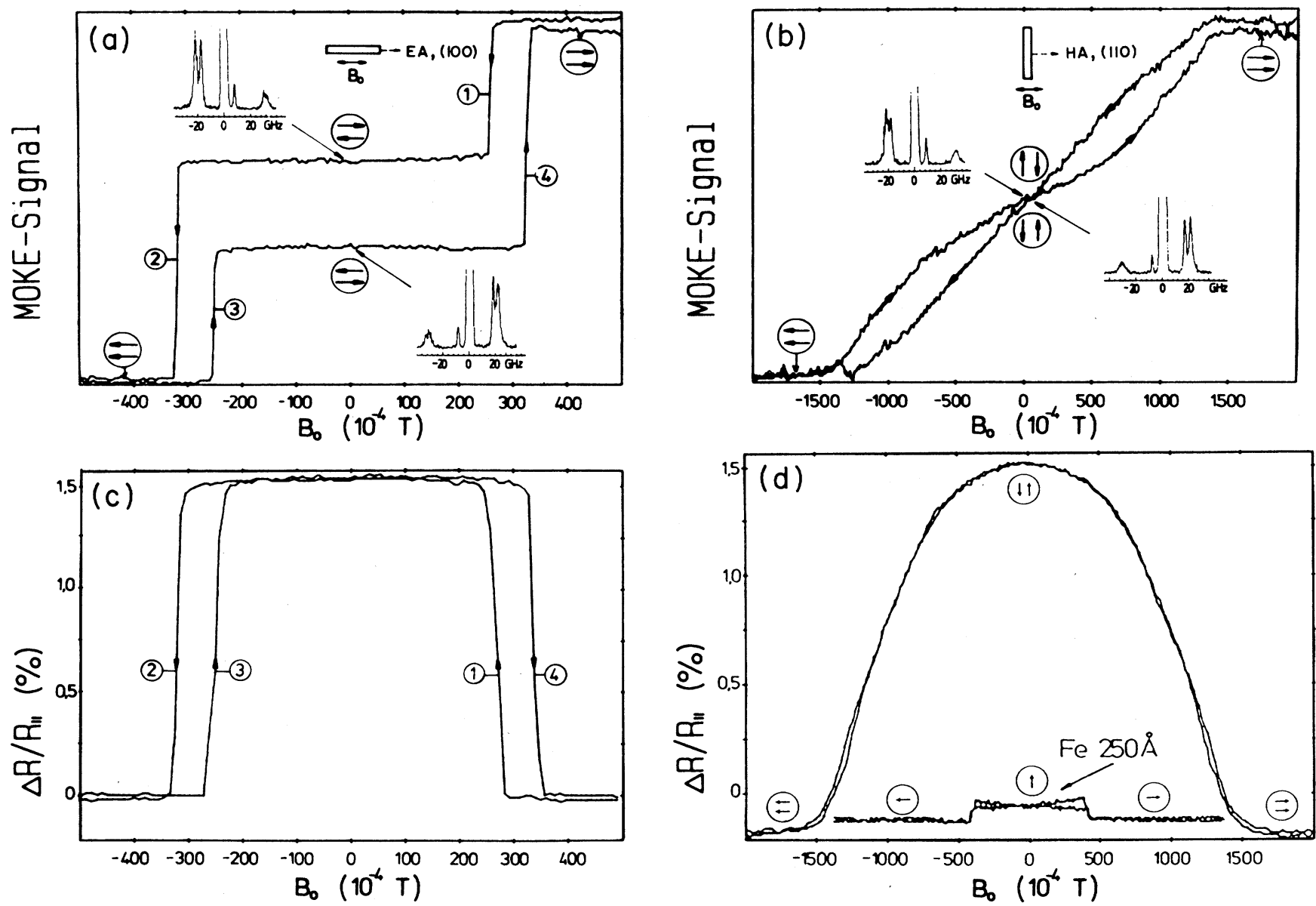

FIG. 2. (a)-(b) MOKE hysteresis curves and (c)-(d) magnetoresistance $\Delta R / R_{\|}=\left(R-R_{\|}\right) / R_{\|}$from Fe double layers with antiferromagnetic coupling. Also, (d) displays the anisotropic MR effect of a 250 - $\AA$-thick Fe film. 
tail elsewhere, ${ }^{7}$ this has to do with the absence of twofold rotational symmetry of the sample displayed in Fig. 1 around a vertical axis. Then waves with positive and negative $k_{\|}$are not linked by symmetry and occur at different frequencies. This is sometimes referred to as nonreciprocal behavior. (For parallel alignment, the twofold rotational symmetry around a vertical axis would be present and Stokes and anti-Stokes scattering would occur symmetric to the elastic scattering.) We see that for the two directions of the field scan the asymmetry reverses. This is linked with the fact that the magnetizations in the single films are reversed correspondingly, supporting the information already obtained from the MOKE intensities. From the scattering geometry we find, in addition, that the magnetization always stays aligned collinear with the external field, because for these spectra $B_{0}$ was perpendicular to the plane of incidence shown in Fig. 1. Opposite to this, for the spectra in Fig. 2(b), the external field was applied parallel to the plane of incidence. Hence for small $B_{0}$ we now have the situation that $J$ is perpendicular to $B_{0}$. We would like to stress that it is important here to be sure about the directions of the magnetizations in the two $\mathrm{Fe}$ films because we want to distinguish between magnetoresistance due to the anisotropic effect and due to antiparallel alignment.

We can now turn to the discussion of the magnetoresistance (MR) traces as shown in Figs. 2(c) and 2(d). Here we have plotted the relative change of the resistivity $\left(R-R_{\|}\right) / R_{\|}$as we scan through the hysteresis loop. $R_{\|}$is the resistivity for saturation along the EA. In Fig. 2(d) we also show the MR of the single Fe film, taken for $B_{0}$ along the HA. Since for large $B_{0}$ the magnetization is along [110] and for small $B_{0}$ along [100], the maximum change of $R$ seen in this trace displays the normal anisotropic MR $\left(R_{\perp}-R_{\|}\right) / R_{\|}$of a $25-\mathrm{nm}$-thick Fe film. The value of $-0.13 \%$ is in reasonable agreement with the literature value of $-0.2 \%{ }^{8}$ For the single film and $B_{0}$ along the EA there was no measurable MR effect. The reason for this is clear; $J$ always stays aligned parallel to the EA and magnetization reversal takes place via domain-wall motion only. In Fig. 2(d) for the Fe-Cr-Fe film, we have an MR effect both due to the anisotropic effect (negative values) and antiparallel alignment (positive values). The zero level of this plot is defined by $R=R_{\|}$. As compared to the single film, the anisotropic effect is increased. We believe that this is due to scattering of electrons in the $\mathrm{Cr}$ interlayer, which is similar to scattering at $\mathrm{Cr}$ impurities in $\mathrm{Fe}-\mathrm{Cr}$ alloys.

The experimental results reported here show that the antiparallel alignment of the magnetizations in our double layers produces an appreciable increase of the electrical resistivity. We propose that this is caused by spin-flip scattering. Electrons in one Fe film will be spin polarized along the direction of $J$. If they penetrate the other film they will find the reversed magnetization direction and undergo spin-flip scattering. We can also describe the situation in a picture of spin-split bands where the role of spinup and spin-down electrons is reversed in the two ferromagnetic films. The states at the Fermi level have oppositely aligned spins in the two antiparallel films, and, accordingly, the conduction electrons have to flip the spin when they pass from one Fe film to the other. This picture in fact tells us that $\mathrm{Fe}$ is probably not even the best candidate for the observed effect because of the overlap of spin-up and spin-down bands. ${ }^{9}$

We also have tested the possibility of a further increase of the effect by fabricating layered structures with more than two Fe films. In Fe trilayers with $d=8 \mathrm{~nm}$ and $d_{0}=1 \mathrm{~nm}$ we obtained $3 \%$, which doubles the effect seen in Fig. 2(d) from the double layers. Further increase to above $10 \%$ has been observed by cooling this sample to about $5 \mathrm{~K}$.

For a quantitative analysis it would be important to know the mean free path for spin-flip scattering. It should be possible to obtain this information from the dependence of the effect on the thickness of the Fe films. Corresponding experiments are currently under way.

Note added. After submission of this manuscript it came to our knowledge that Baibich et al. have observed magnetoresistance changes as high as $50 \%$ at low temperatures in multilayered $\mathrm{Fe}-\mathrm{Cr}$ structures. ${ }^{10}$

We want to acknowledge the skillful assistance of $R$. Schreiber during sample preparation.
${ }^{1}$ P. Grünberg, R. Schreiber, Y. Pang, M. B. Brodsky, and H. Sowers, Phys. Rev. Lett. 57, 2442 (1986).

${ }^{2}$ P. Grünberg, R. Schreiber, Y. Pang, U. Walz, M. B. Brodsky, and H. Sowers, J. Appl. Phys. 61, 3750 (1987).

${ }^{3}$ F. Saurenbach, U. Walz, L. Hinchey, P. Gruenberg, and W. Zinn, J. Appl. Phys. 63, 3473 (1988).

${ }^{4} \mathrm{P}$. Grünberg, in Thin Film Growth Techniques For Low Dimensional Structures, edited by R. F. C. Farrow, S. S. P. Parkin, P. J. Dobson, J. H. Neave, and A. S. Arrott (Plenum, New York, 1987).

${ }^{5}$ G. A. Prinz and J. J. Krebs, Appl. Phys. Lett. 39, 397 (1981).
${ }^{6}$ G. A. Prinz, G. T. Rado, and J. J. Krebs, J. Appl. Phys. 53, 2087 (1982).

${ }^{7}$ K. Mika and P. Grünberg, Phys. Rev. B 31, 4465 (1985).

${ }^{8}$ R. T. McGuire and R. I. Potter, IEEE Trans. Magn. 11, 1018 (1975).

${ }^{9}$ A. R. Williams, V. L. Moruzzi, A. P. Malozemoff, and K. Terakura, IEEE Trans. Magn. 19, 5 (1983).

${ }^{10}$ M. N. Baibich, J. M. Broto, A. Fert, F. Nguyen Van Dau, F. Petroff, P. Etienne, G. Creuzet, A. Friedrich, and J. Chazelas, Phys. Rev. Lett. 61, 2472 (1988). 